\title{
The Effects of Stress Tensor Fluctuations upon Focusing
}

\author{
J. Borgman and L.H. Ford* \\ Institute of Cosmology \\ Department of Physics and Astronomy \\ Tufts University, Medford, MA 02155
}

\begin{abstract}
We treat the gravitational effects of quantum stress tensor fluctuations. An operational approach is adopted in which these fluctuations produce fluctuations in the focusing of a bundle of geodesics. This can be calculated explicitly using the Raychaudhuri equation as a Langevin equation. The physical manifestation of these fluctuations are angular blurring and luminosity fluctuations of the images of distant sources. We give explicit results for the case of a scalar field on a flat background in a thermal state.
\end{abstract}

PACS numbers: 04.62.+v, 04.60.-m, 05.40.-a

*Email: ford@cosmos.phy.tufts.edu 


\section{INTRODUCTION}

Although quantum states in field theory may or may not be eigenstates of the Hamiltonian, and hence have fixed energy, they are never eigenstates of the energy density or other stress tensor components. This means that all quantum field theories exhibit fluctuations of the stress tensor. These fluctuations can manifest themselves either through fluctuating forces on material bodies, or through fluctuations of the gravitational field. In the former category are fluctuations of Casimir forces [1, 2, 3, 4] and fluctuations of the radiation pressure [5]. In this paper, we will be concerned with the latter phenomenon, where stress tensor fluctuations drive fluctuations of the spacetime geometry. These are "passive" fluctuations of the spacetime, in contrast to the "active" fluctuations which arise from the quantization of gravity itself. The role of stress tensor fluctuations in gravity theory has been discussed by several authors 6 , 7, 8, 9, 10, 11, 12, 13, 14, 15, 16, 17]. Among the issues of interest is the extent to which these fluctuations place limits on the validity of the semiclassical theory of gravity, which is the approximation in which the gravitational field is assumed to be classical and determined by the renormalized expectation value of the stress tensor [6, 8, 16]. Other authors have been concerned with the possible role of stress tensor fluctuations in the early universe or upon black hole evaporation [10, 11, 12, 13, 14, 15].

One of the basic problems which must be addressed in treatments of quantum stress tensor fluctuations is that the stress tensor correlation function is singular, even if the stress tensor operator has been renormalized to have finite expectation values. For this reason, many of the earlier treatments [6, 7, 8, 9, 14] studied only a portion of the correlation function which is free of singularities, the "fully normal ordered part". However, more recently it has become clear that the remaining parts (the "vacuum" and "cross" terms to be defined in Sect. III) can also have physical effects, and that extracting these effects requires a careful procedure for defining integrals with singular integrands [5, 17]. For example, the radiation pressure fluctuations, arising when light in a coherent state impinges upon a mirror, come entirely from the cross term [5].

In this paper, we will present an approach to studying the gravitational effects of stress tensor fluctuations which is based upon Raychaudhuri's equation. This equation relates the Ricci tensor to the rate of change of the expansion along a congruence of geodesics, and plays a central role in the proofs of the classical singularity theorems. We will treat it as a Langevin equation which describes how stress tensor fluctuations lead to fluctuations in the expansion. This in turn leads to a lack of focusing and fluctuations in the luminosity of the images of distant objects seen through a fluctuating spacetime geometry. Here the quantum matter field will be a massless, minimally coupled scalar field on an average background of Minkowski spacetime. The outline of the paper is as follows: Section III will discuss the stress tensor correlation function and its decomposition into individual terms. Section III will deal with the Ricci tensor correlation function and the fluctuations of the expansion. The treatment of the singular pieces in the correlation function will also be discussed in this section. In Sec. IV] we will explicitly calculate the expansion fluctuations for a thermal state. The results of the paper will be summarized and discussed in Sec. $\nabla$ Except as otherwise noted, we use units in which $\hbar=c=G=k=1$, where $G$ and 
$k$ are Newton's and Boltzmann's constants, respectively.

\section{QUANTUM STRESS TENSOR FLUCTUATIONS}

In this paper, we will deal with the fluctuations of the stress tensor for quantum fields on an average background of Minkowski spacetime. The formal expectation values of $T_{\mu \nu}$ are divergent, so we renormalize by subtracting the expectation value in the Minkowski vacuum state:

$$
: T_{\mu \nu}:=T_{\mu \nu}-\left\langle T_{\mu \nu}\right\rangle_{0},
$$

where $: T_{\mu \nu}:$ is the normal ordered stress tensor operator. The fluctuations of $: T_{\mu \nu}$ : are described by the stress tensor correlation function

$$
S_{\mu \nu \alpha \beta}\left(x, x^{\prime}\right)=\left\langle: T_{\mu \nu}(x):: T_{\alpha \beta}\left(x^{\prime}\right):\right\rangle-\left\langle: T_{\mu \nu}(x):\right\rangle\left\langle: T_{\alpha \beta}\left(x^{\prime}\right):\right\rangle .
$$

Here the expectation value can be taken in an arbitrary quantum state. It is of interest to note that this correlation function is unchanged when $: T_{\mu \nu}$ : is shifted by a constant, so we could formally write $S_{\mu \nu \alpha \beta}\left(x, x^{\prime}\right)$ without normal ordering:

$$
S_{\mu \nu \alpha \beta}\left(x, x^{\prime}\right)=\left\langle T_{\mu \nu}(x) T_{\alpha \beta}\left(x^{\prime}\right)\right\rangle-\left\langle T_{\mu \nu}(x)\right\rangle\left\langle T_{\alpha \beta}\left(x^{\prime}\right)\right\rangle .
$$

Although $\left\langle: T_{\mu \nu}(x):\right\rangle$ is finite in all well behaved quantum states, $S_{\mu \nu \alpha \beta}\left(x, x^{\prime}\right)$ is singular in the limit that $x$ and $x^{\prime}$ are null separated. There is a useful decomposition of operator products which classifies the various contributions to the correlation function. Let $T(x)=: \phi_{1}(x) \phi_{2}(x)$ :, where $\phi_{1}$ and $\phi_{2}$ are free quantum fields, or derivatives of fields. The stress tensor operator $: T_{\mu \nu}$ : is a sum of such terms. It may be shown using Wick's theorem that

$$
T(x) T\left(x^{\prime}\right)=S_{0}+S_{1}+S_{2},
$$

where

$$
\begin{aligned}
S_{0} & =\left\langle\phi_{1}(x) \phi_{1}\left(x^{\prime}\right)\right\rangle_{0}\left\langle\phi_{2}(x) \phi_{2}\left(x^{\prime}\right)\right\rangle_{0}+\left\langle\phi_{1}(x) \phi_{2}\left(x^{\prime}\right)\right\rangle_{0}\left\langle\phi_{2}(x) \phi_{1}\left(x^{\prime}\right)\right\rangle_{0}, \\
S_{1} & =: \phi_{1}(x) \phi_{1}\left(x^{\prime}\right):\left\langle\phi_{2}(x) \phi_{2}\left(x^{\prime}\right)\right\rangle_{0}+: \phi_{1}(x) \phi_{2}\left(x^{\prime}\right):\left\langle\phi_{2}(x) \phi_{1}\left(x^{\prime}\right)\right\rangle_{0} \\
& +: \phi_{2}(x) \phi_{1}\left(x^{\prime}\right):\left\langle\phi_{1}(x) \phi_{2}\left(x^{\prime}\right)\right\rangle_{0}+: \phi_{2}(x) \phi_{2}\left(x^{\prime}\right):\left\langle\phi_{1}(x) \phi_{1}\left(x^{\prime}\right)\right\rangle_{0},
\end{aligned}
$$

and

$$
S_{2}=: \phi_{1}(x) \phi_{2}(x) \phi_{1}\left(x^{\prime}\right) \phi_{2}\left(x^{\prime}\right):
$$

Thus the operator product $T(x) T\left(x^{\prime}\right)$ consists of a purely vacuum part $S_{0}$, a fully normal-ordered part $S_{2}$, and a part $S_{1}$ which is a cross term between the vacuum and normal-ordered parts. 
The expectation value of the product of stress tensors can be expressed as the sum of these three contributions:

$$
\begin{aligned}
\left\langle: T_{\mu \nu}(x) T_{\alpha \beta}\left(x^{\prime}\right):\right\rangle & =\left\langle: T_{\mu \nu}(x):: T_{\alpha \beta}\left(x^{\prime}\right):\right\rangle+\left\langle T_{\mu \nu}(x) T_{\alpha \beta}\left(x^{\prime}\right)\right\rangle_{\text {cross }} \\
& +\left\langle T_{\mu \nu}(x) T_{\alpha \beta}\left(x^{\prime}\right)\right\rangle_{v a c},
\end{aligned}
$$

where the last two terms are the cross term and vacuum term, respectively. In the limit that $x^{\prime} \rightarrow x$,

$$
\left\langle T_{\mu \nu}(x) T_{\alpha \beta}\left(x^{\prime}\right)\right\rangle_{\text {cross }} \sim \frac{1}{\left(x-x^{\prime}\right)^{4}},
$$

and

$$
\left\langle T_{\mu \nu}(x) T_{\alpha \beta}\left(x^{\prime}\right)\right\rangle_{v a c} \sim \frac{1}{\left(x-x^{\prime}\right)^{8}} .
$$

Observable quantities will be expressed as integrals of the correlation function. However, the singularities in the cross and vacuum terms are not integrable in a naive way. As discussed in Refs. [4, 5, 17], one solution to this problem is an integration by parts procedure. This method allows one to define the relevant integrals, and will be discussed in more detail below. An alternative approach, which will not be pursued in detail in this paper, is to use dimensional regularization. In this approach, the divergent part of a stress tensor correlation function can be expressed in a local form, which arises from counterterms in the action of the form of $R^{2}$ and $R_{\mu \nu} R^{\mu \nu}$, where $R_{\mu \nu}$ is the Ricci tensor [18].

\section{RICCI TENSOR FLUCTUATIONS AND RAYCHAUDHURI'S EQUA-}

\section{TION}

\section{A. Raychaudhuri's Equation as a Langevin Equation}

For a classical spacetime, Einstein's equations relate the Ricci tensor $R_{\mu \nu}$ to the stress tensor by

$$
R_{\mu \nu}=8 \pi\left(T_{\mu \nu}-\frac{1}{2} g_{\mu \nu} T_{\rho}^{\rho}\right)
$$

In the case of a spacetime with fluctuations driven by stress tensor fluctuations, we assume that this algebraic relation between the Ricci and stress tensors still holds. A useful probe of the fluctuations of the Ricci tensor is the Raychaudhuri equation.

Consider a congruence of either timelike or null geodesics with affine parameter $\lambda$ and tangent vector field $k^{\mu}$. The Raychaudhuri equation gives the rate of change of the expansion $\theta$ along the congruence to be [19]

$$
\frac{d \theta}{d \lambda}=-R_{\mu \nu} k^{\mu} k^{\nu}-a \theta^{2}-\sigma_{\mu \nu} \sigma^{\mu \nu}+\omega_{\mu \nu} \omega^{\mu \nu} .
$$

Here $\sigma^{\mu \nu}$ is the shear and $\omega^{\mu \nu}$ is the vorticity of the congruence. The constant $a=1 / 2$ for null geodesics, and $a=1 / 3$ for timelike geodesics. We are interested in 
interpreting this equation as a Langevin equation in which the Ricci tensor fluctuates. For the purposes of this paper, we will further assume that the shear and vorticity of the congruence vanishes, and that the expansion remains sufficiently small that the $\theta^{2}$ term can also be ignored, so we can write

$$
\frac{d \theta}{d \lambda}=-R_{\mu \nu} k^{\mu} k^{\nu}
$$

where $R_{\mu \nu}$ is determined by the fluctuating stress tensor by Eq. (11).

Let the Ricci tensor correlation function be defined to be

$$
C_{\mu \nu \alpha \beta}\left(x, x^{\prime}\right)=\left\langle R_{\mu \nu}(x) R_{\alpha \beta}\left(x^{\prime}\right)\right\rangle-\left\langle R_{\mu \nu}(x)\right\rangle\left\langle R_{\alpha \beta}\left(x^{\prime}\right)\right\rangle .
$$

The variance of the expansion can be expressed as a double integral of this correlation function as

$$
\left\langle\theta^{2}\right\rangle-\langle\theta\rangle^{2}=\left\langle(\Delta \theta)^{2}\right\rangle=\int_{0}^{\lambda_{0}} d \lambda \int_{0}^{\lambda_{0}} d \lambda^{\prime} C_{\mu \nu \alpha \beta}\left(\lambda, \lambda^{\prime}\right) k^{\mu}(\lambda) k^{\nu}(\lambda) k^{\alpha}\left(\lambda^{\prime}\right) k^{\beta}\left(\lambda^{\prime}\right) .
$$

Here we are assuming that the fluctuations of the expansion occur in a finite affine parameter interval, between $\lambda=0$ and $\lambda=\lambda_{0}$. In the case of a flat background, we can take the stress tensor to be normal ordered. Then the Ricci tensor correlation function $C_{\mu \nu \alpha \beta}\left(x, x^{\prime}\right)$ is related to the correlation function for the normal ordered stress tensor by

$$
C_{\mu \nu \alpha \beta}\left(x, x^{\prime}\right)=64 \pi^{2}\left(S_{\mu \nu \alpha \beta}-\frac{1}{2} g_{\mu \nu} S_{\rho \alpha \beta}^{\rho}-\frac{1}{2} g_{\alpha \beta} S_{\mu \nu \rho}^{\rho}+\frac{1}{4} g_{\mu \nu} g_{\alpha \beta} S_{\rho}^{\rho \sigma} \sigma\right),
$$

and can also be decomposed into fully normal ordered, cross, and vacuum terms.

In the remainder of this paper, we will take the matter field to be a massless, minimally coupled scalar field, for which the stress tensor is

$$
T_{\mu \nu}=\phi_{, \mu} \phi_{, \nu}-\frac{1}{2} g_{\mu \nu} \phi^{, \rho} \phi_{, \rho}
$$

and the Ricci tensor is

$$
R_{\mu \nu}=8 \pi \phi_{, \mu} \phi_{, \nu}
$$

This will now be interpreted as a normal ordered operator, so the correlation function $C_{\mu \nu \alpha \beta}\left(x, x^{\prime}\right)$ can be expressed as a sum of a fully normal ordered term, a cross term, and a vacuum term:

$$
C^{\mu \nu \alpha \beta}\left(x, x^{\prime}\right)=C^{\mu \nu \alpha \beta}\left(x, x^{\prime}\right)_{N O}+C^{\mu \nu \alpha \beta}\left(x, x^{\prime}\right)_{c r o s s}+C^{\mu \nu \alpha \beta}\left(x, x^{\prime}\right)_{v a c} .
$$

Each of these terms can be found explicitly from Eqs. (15), (66), and (77) to be

$$
\begin{aligned}
C^{\mu \nu \alpha \beta}\left(x, x^{\prime}\right)_{N O} & =64 \pi^{2}\left\langle: \partial^{\mu} \phi(x) \partial^{\nu} \phi(x) \partial^{\prime \alpha} \phi\left(x^{\prime}\right) \partial^{\prime \beta} \phi\left(x^{\prime}\right):\right\rangle \\
C^{\mu \nu \alpha \beta}\left(x, x^{\prime}\right)_{c r o s s} & =64 \pi^{2}\left(\partial^{\mu} \partial^{\prime \alpha} D \partial^{\nu} \partial^{\prime \beta} D_{0}+\partial^{\nu} \partial^{\prime \alpha} D \partial^{\mu} \partial^{\prime \beta} D_{0}\right. \\
& \left.+\partial^{\nu} \partial^{\prime \beta} D \partial^{\mu} \partial^{\prime \alpha} D_{0}+\partial^{\mu} \partial^{\prime \beta} D \partial^{\nu} \partial^{\prime \alpha} D_{0}\right)
\end{aligned}
$$


and

$$
C^{\mu \nu \alpha \beta}\left(x, x^{\prime}\right)_{v a c}=64 \pi^{2}\left(\partial^{\mu} \partial^{\prime \alpha} D_{0} \partial^{\nu} \partial^{\prime \beta} D_{0}+\partial^{\nu} \partial^{\prime \alpha} D_{0} \partial^{\mu} \partial^{\prime \beta} D_{0}\right) .
$$

Here $\partial^{\mu}$ denotes differentiation at point $x$ and $\partial^{\prime \alpha}$ that at $x^{\prime}$. The vacuum two-point function is

$$
D_{0}=\left\langle\phi(x) \phi\left(x^{\prime}\right)\right\rangle_{0}=\frac{1}{4 \pi^{2}\left(x-x^{\prime}\right)^{2}},
$$

and

$$
D=\left\langle: \phi(x) \phi\left(x^{\prime}\right):\right\rangle
$$

is the renormalized two-point function in the chosen state.

The central quantity which we calculate is the variance of the expansion parameter, $\left\langle(\Delta \theta)^{2}\right\rangle$. However, it will be useful to relate it to more directly observable quantities. Two possibilities are characteristic angle by which an images will be blurred by metric fluctuations, $\Delta \varphi$, and the fractional fluctuations in the apparent luminosity of the source, $\Delta L / L$. In Appendix A, we give a heuristic treatment of the relation between $\Delta \varphi$ and $\Delta \theta$, and argue that we should expect a blurring angle of the order of $\Delta \varphi=s \Delta \theta / 2$, where $s$ is the distance to the source. Our treatment is heuristic because we actually analyze the situation of a source in flat spacetime whose position fluctuates. We conjecture that a careful treatment of the effects of a fluctuating spacetime geometry will give a result of the same order of magnitude. Fluctuations of $\theta$ are also related to fluctuations in the brightness, or apparent luminosity of the source, as is discussed in Appendix B. In general, $\Delta L / L$ is given in terms of an integral of the expansion correlation function, however, in some cases, it is of the same order as $\Delta \varphi$.

\section{B. Averaging over a Bundle of Geodesics}

In order for the contributions of the cross and vacuum terms to be finite, it is necessary to average over a spacetime volume. This volume is the interior of the world tube defined by the bundle of geodesics. Thus in Eq. (15), we replace the integrations on the affine parameter by four-dimensional spacetime integrations and write

$$
\left\langle(\Delta \theta)^{2}\right\rangle=\int d^{4} x \int d^{4} x^{\prime \prime} f(x) f\left(x^{\prime}\right) C_{\mu \nu \alpha \beta}\left(x, x^{\prime}\right) k^{\mu}(x) k^{\nu}(x) k^{\alpha}\left(x^{\prime}\right) k^{\beta}\left(x^{\prime}\right) .
$$

where $f(x)$ is the function which describes the shape of the world tube.

It is convenient to express this averaging in null coordinates. Let the unperturbed rays propagate in the $x$-direction, and let $u=t-x$ and $v=t+x$ be the null coordinates. We can take the affine parameter to be the $v$ - coordinate, and then introduce additional averaging in the $u, y$, and $z$ directions. We will take the averaging functions to be Lorenztian or Gaussian functions in each of these directions. Let

$$
g_{L}(u, \alpha)=\frac{\alpha}{\pi\left(u^{2}+\alpha^{2}\right)},
$$


and

$$
g_{G}(u, \alpha)=\frac{1}{\sqrt{\pi} \alpha} \mathrm{e}^{-u^{2} / \alpha^{2}}
$$

so that

$$
\int_{-\infty}^{\infty} g_{L}(u, \alpha) d u=\int_{-\infty}^{\infty} g_{G}(u, \alpha) d u=1
$$

Equation (25) involves an averaging in six variables, $u, u^{\prime}, y, y^{\prime}, z$, and $z^{\prime}$, as well as integrations over $v$ and $v^{\prime}$. However, in the cases that will be of interest, the correlation function depends only upon the differences $\Delta u=u-u^{\prime}$, etc. We can exploit the property of Lorenztian and Gaussian functions that averaging in $u$ and $u^{\prime}$ separately is equivalent to an average on $\Delta u$. In particular,

$$
\int_{-\infty}^{\infty} d u \int_{-\infty}^{\infty} d u^{\prime} g_{L}(u, \alpha) g_{L}\left(u^{\prime}, \alpha\right) F\left(u-u^{\prime}\right)=\int_{-\infty}^{\infty} d \Delta u g_{L}(\Delta u, 2 \alpha) F(\Delta u)
$$

and

$$
\int_{-\infty}^{\infty} d u \int_{-\infty}^{\infty} d u^{\prime} g_{G}(u, \alpha) g_{G}\left(u^{\prime}, \alpha\right) F\left(u-u^{\prime}\right)=\int_{-\infty}^{\infty} d \Delta u g_{G}(\Delta u, \sqrt{2} \alpha) F(\Delta u) .
$$

Then we need only average over the differences $\Delta u, \Delta y$, and $\Delta z$. It will be convenient to let the averaging function be a Lorenztian of width $a$ in $\Delta u$ and Gaussians of width $b$ in each of $\Delta y$ and $\Delta z$, so

$$
f(x)=g_{L}(\Delta u, a) g_{G}(\Delta y, b) g_{G}(\Delta z, b) .
$$

The integrations on $v$ and $v^{\prime}$ already amount to averaging, so we do not need to introduce any additional smearing in these variables. If the integrand is a function of the difference $\Delta v=v-v^{\prime}$ then we may use the relation

$$
\int_{0}^{v_{0}} d v \int_{0}^{v_{0}} d v^{\prime} F\left(v-v^{\prime}\right)=\int_{0}^{v_{0}} d \Delta v\left(v_{0}-\Delta v\right) F(\Delta v)+\int_{-v_{0}}^{0} d \Delta v\left(v_{0}+\Delta v\right) F(\Delta v) .
$$

If $F(\Delta v)$ is an even function, this relation becomes

$$
\int_{0}^{v_{0}} d v \int_{0}^{v_{0}} d v^{\prime} F\left(v-v^{\prime}\right)=2 \int_{0}^{v_{0}} d \Delta v\left(v_{0}-\Delta v\right) F(\Delta v)
$$

Henceforth, we will slightly change the notation, dropping the " $\Delta$ "s, and write $v, u, y$, and $z$ for $\Delta v, \Delta u, \Delta y$, and $\Delta z$, respectively. Now our expression for the averaged variance in the expansion becomes

$$
\begin{aligned}
& \left\langle(\Delta \theta)^{2}\right\rangle=\left[\int_{0}^{v_{0}} d v\left(v_{0}-v\right)+\int_{-v_{0}}^{0} d v\left(v_{0}+v\right)\right] \int_{-\infty}^{\infty} d u g_{L}(u, a) \times \\
& \int_{-\infty}^{\infty} d y \int_{-\infty}^{\infty} d z g_{G}(y, b) g_{G}(z, b) C_{\mu \nu \alpha \beta}\left(x, x^{\prime}\right) k^{\mu}(x) k^{\nu}(x) k^{\alpha}\left(x^{\prime}\right) k^{\beta}\left(x^{\prime}\right) .
\end{aligned}
$$




\section{Analysis of the Cross and Vacuum Terms}

Here we wish to look at the state-independent and potentially singular factors in the cross and the vacuum terms. Let

$$
\sigma=\frac{1}{2}\left(x-x^{\prime}\right)^{2}=\frac{1}{2} \Delta x^{\mu} \Delta x_{\mu} .
$$

Then the vacuum two-point function is

$$
D_{0}(\sigma)=\frac{1}{8 \pi^{2} \sigma}
$$

Further let

$$
B \equiv k_{\mu} k_{\alpha} \partial^{\mu} \partial^{\prime \alpha} D_{0}=-\left(k_{\mu} k^{\mu}\right) D_{0}^{\prime}-\left(k_{\mu} \Delta x^{\mu}\right)^{2} D_{0}^{\prime \prime} .
$$

Here $D_{0}^{\prime}$ and $D_{0}^{\prime \prime}$ are derivatives of $D_{0}$ with respect to $\sigma$. The contribution of the vacuum term to $C_{\mu \nu \alpha \beta} k^{\mu} k^{\nu} k^{\alpha} k^{\beta}$, the integrand in Eq. (34), is

$$
C_{\mathrm{vac}}^{\mu \nu \alpha \beta} k_{\mu} k_{\nu} k_{\alpha} k_{\beta}=128 \pi^{2} B^{2} \text {. }
$$

Similarly, the contribution of the cross term is

$$
C_{\text {cross }}^{\mu \nu \alpha \beta} k_{\mu} k_{\nu} k_{\alpha} k_{\beta}=256 \pi^{2} k_{\mu} k_{\alpha}\left(\partial^{\mu} \partial^{\prime \alpha} D\right) B
$$

The contributions of these terms must be defined by averaging, as in Eq. (25). However, we can take the tangent vector $k^{\mu}$ to be null, even under the averaging, so all of the rays in the bundle over which we average have the same direction. Then $k^{\mu} k_{\mu}=0$ and the first term in $B$ vanishes. We can take $k_{\mu}=-\delta_{\mu}^{u}$. then

$$
B=-\frac{2 u^{2}}{\pi^{2}\left(r^{2}-u v\right)^{3}},
$$

where $r^{2}=y^{2}+z^{2}$. The vacuum contribution to $\left\langle(\Delta \theta)^{2}\right\rangle$ becomes

$$
\begin{aligned}
& \left\langle(\Delta \theta)^{2}\right\rangle_{v a c}=\frac{1024}{\pi^{2}} \int_{0}^{v_{0}} d v\left(v_{0}-v\right) \times \\
& \int_{-\infty}^{\infty} d u \int_{-\infty}^{\infty} d y \int_{-\infty}^{\infty} d z g_{L}(u, a) g_{G}(y, b) g_{G}(z, b)\left[\frac{u^{4}}{\pi^{2}\left(r^{2}-u v\right)^{6}}\right] .
\end{aligned}
$$

Note that once the integration on $u$ is performed, the integrand is an even function of $v$, so we can use Eq. (33).

Let us first consider the $u$-integration. The integrand has simple poles at $u= \pm i a$, coming from the sampling function $g_{L}(u, a)$. However, it also has a sixth-order pole at $u=r^{2} / v$, which reflects the singular nature of the vacuum term. As is well-known, contour integrals around higher order poles may be evaluated by integration by parts. Here the answer must be a real quantity, so we can write the integral on $u$ as the average of two integrals, one over a contour closed in the upper-half plane and going above the sixth-order pole on the real axis, and a second over a contour closed in the 
lower-half plane and going below this pole. The result is finite and just the real part of the residue of the pole at either $u=i a$ or $u=-i a$,

$$
\int_{-\infty}^{\infty} d u g_{L}(u, a)\left[\frac{u^{4}}{\left(r^{2}-u v\right)^{6}}\right]=\operatorname{Re}\left[\frac{a^{4}}{\left(r^{2}-i a v\right)^{6}}\right]
$$

It is convenient to perform the $v$-integration next, which yields

$$
\int_{0}^{v_{0}} d v\left(v_{0}-v\right) \operatorname{Re}\left[\frac{a^{4}}{\left(r^{2}-i a v\right)^{6}}\right]=\frac{a^{4} v_{0}^{2}\left(a^{6} v_{0}^{6}+4 a^{4} v_{0}^{4} r^{4}+5 a^{2} v_{0}^{2} r^{8}+10 r^{12}\right)}{20 r^{8}\left(a^{2} v_{0}^{2}+r^{4}\right)^{4}}
$$

In the limit that $v_{0} \gg r^{2} / a$, this approaches $a^{2} /\left(20 r^{8}\right)$. Both the exact form, Eq. (43), and the large $v_{0}$ limit have the same $r^{-8}$ behavior for small $r$. In either case, a further averaging in the transverse directions is needed to produce a finite result.

In the large $v_{0}$ limit, we can write

$$
\left\langle(\Delta \theta)^{2}\right\rangle_{v a c}=\frac{256}{5 \pi^{2}} a^{2}\left\langle\frac{1}{r^{8}}\right\rangle
$$

The evaluation of quantities such as $\left\langle 1 / r^{8}\right\rangle$ is discussed in Appendix C, where it is argued that

$$
\left\langle\frac{1}{r^{8}}\right\rangle=\frac{A_{8}}{b^{8}}
$$

where $A_{8}$ has at most a logarithmic dependence on $b$. This leads to our final result for the contribution of the vacuum term in the large $v_{0}$ limit,

$$
\left\langle(\Delta \theta)^{2}\right\rangle_{v a c}=\ell_{P}^{4} \frac{256 A_{8} a^{2}}{5 \pi^{2} b^{8}}
$$

where $\ell_{P}$ is the Planck length.

Note that $v_{0}=2 s$, where $s$ is the spatial separation between the source and detector, and that the large $v_{0}$ limit now means $v_{0} \gg b^{2} / a$. The fact that $\left\langle(\Delta \theta)^{2}\right\rangle_{v a c}$ approaches a finite value in this limit indicates that the focusing fluctuations in the Minkowski vacuum do not accumulate, but they can be non-zero. This raises the question as to whether they lead to potentially observable effects. The expected angular blurring or fractional luminosity fluctuations of an image at a distance $s$ are given by Eqs. (A12) and (B9), respectively, and in this case will be

$$
2 \Delta \varphi=\left(\frac{\Delta L}{L}\right)_{r m s}=0.1 A_{8} \ell_{P}^{2} \frac{a s}{b^{4}} \approx 10^{-8} A_{8}\left(\frac{a}{b}\right)\left(\frac{10^{-10} \mathrm{~cm}}{b}\right)^{3}\left(\frac{s}{10^{28} \mathrm{~cm}}\right) .
$$

The smallest reasonable averaging scale would be of the order of the wavelength of the photons from the source. Even in this case, the effect predicted by Eq. (47) is too small to have been seen in any measurements that have yet been performed.

It is also of interest to note that the asymptotic value of $\left\langle(\Delta \theta)^{2}\right\rangle_{v a c}$ can be made arbitrarily small by making the temporal averaging scale $a$ small, although the value of $v_{0}$ required to attain this asymptotic value grows. This behavior can be traced to 
the factor of $\left(k_{\mu} \Delta x^{\mu}\right)^{4}$ in Eq. (38). This factor in turn contributes a factor of $a^{4}$ to $\left\langle(\Delta \theta)^{2}\right\rangle_{v a c}$, and turns what would otherwise be an $a^{-2}$ dependence into $a^{2}$ dependence. A general product of stress tensor operators needs to be averaged in time to be finite, but the particular combination that contribute to $\left\langle(\Delta \theta)^{2}\right\rangle_{v a c}$ remains finite even in the limit that the time averaging scale goes to zero.

Now let us turn to the cross term, which is determined by Eq. (39). This term also requires averaging to render it finite. However, it is state-dependent, so we cannot calculate it explicitly without specifying the function $D$. However, we can look at the result of averaging the state-independent factor $B$, and then assuming that the state-dependent factor is approximately independent of $v$. This leads to

$$
\left\langle(\Delta \theta)^{2}\right\rangle_{\text {cross }}=512 \pi^{2} k_{\mu} k_{\alpha}\left(\partial^{\mu} \partial^{\prime \alpha} D\right) \int_{0}^{v_{0}} d v\left(v_{0}-v\right)\langle B\rangle
$$

We may evaluate the integral of $\langle B\rangle$ in the same manner as used for the vacuum term:

$$
\langle B\rangle=\int d^{2} x g(r) \int_{-\infty}^{\infty} d u g_{L}(u, a) B=-\frac{2 a^{2}}{\pi^{2}} \int d^{2} x g(r) \frac{r^{2}\left(3 a^{2} v^{2}-r^{4}\right)}{\left(a^{2} v^{2}+r^{4}\right)^{3}} .
$$

Then

$$
\int_{0}^{v_{0}} d v\left(v_{0}-v\right)\langle B\rangle=\frac{a^{2}}{\pi^{2}} \int d^{2} x g(r) \frac{a^{2} v_{0}^{2}}{\left(a^{2} v_{0}^{2}+r^{4}\right)} .
$$

Note that this expression, and hence the contribution of the cross term, vanishes in the limit that $a \rightarrow 0$ for fixed $v_{0}$. However, if we let $v_{0} \rightarrow \infty$ for fixed, nonzero $a$, then we find

$$
\int_{0}^{v_{0}} d v\left(v_{0}-v\right)\langle B\rangle=\frac{1}{\pi^{2}}\left\langle\frac{1}{r^{2}}\right\rangle=\frac{A_{2}}{\pi^{2} b^{2}},
$$

where $A_{2}$ has at most a logarithmic dependence on $b$. As expected, the cross term is singular in the limit that $b \rightarrow 0$, but less so than the vacuum term.

As was the case for the vacuum term, $\left\langle(\Delta \theta)^{2}\right\rangle_{\text {cross }}$ vanishes in the limit that $a \rightarrow 0$. In both cases, this is due to the presence of the factor of $\left(k_{\mu} \Delta x^{\mu}\right)^{2}$ in $B$. Note that if we were to take first the limit $a \rightarrow 0$ and then the limit $b \rightarrow 0$, we would have a localized ray for which $\left\langle(\Delta \theta)^{2}\right\rangle$ is given entirely by the fully normal ordered term.

Here we have used a specific choice of averaging. One topic for future research will be to generalize this choice. In particular, it will be of interest to write covariant expressions for $B$ and $B^{2}$ as derivatives of logarithmic functions, and then integrate over a four-dimensional spacetime region.

\section{The Physical Meaning of Smearing Scales $a$ and $b$}

We have defined the singular terms of the Ricci tensor by averaging over a world tube containing the bundle of geodesics. The shape of this world tube is determined by its spatial width $b$, which describes the transverse distance from the central geodesic, and its temporal width $a$. 
As far as the temporal smearing is concerned, it is worth noting that the parameter $a$ only appears in the pure vacuum term, and does not affect results obtained for state-dependent terms (the cross terms and the fully normal-ordered term). If one assumes that pure vacuum effects in Minkowski spacetime are unphysical, or least unobservable, a discussion of the meaning of $a$ is rendered superfluous. However, if we remain open to the possibility that the pure vacuum effects possess physical significance, we should attempt to connect $a$ with some concrete length scale in the problem. There are a few reasonable options to chose from. Certainly the following come to mind: the wavelength of the source, the coherence length of the source, and the duration of time over which the light source is collected. The latter, in at least one respect, seems the least appealing. It would suggest that the vacuum effect could be made arbitrarily large by leaving the measuring device on for an arbitrarily long time (noting the power dependence in Eq. (47). If we are concerned with finding a upper bound when the measuring process is arbitrarily extended in time, it seems more likely that the coherence length of the light source, or even the smallest scale involved, the wavelength, should be related to the temporal smearing. The argument here is that the photon extends off the lightcone, but certainly no more than the temporal size of its wave packet, and no less than the resolution provided by its characteristic wavelength.

On the other hand, it seems most natural to associate the width of the bundle with the parameters of the experimental set-up, determined by both conditions at the source and at the observer. That is, it seems we can associate the width of the bundle $b$ with the two quantities: the aperture of the device used to collect the light rays, and the size of the emitting source. If both could be fixed to the same size, we would effectively enclose the bundle of geodesics within a cylinder extending along the entire flight path. Under such a circumstance it seems safe to say that $b$ would simply be the radius of the cylinder. In the case of point source emissions, $b$ might be sensibly set to the average width of the bundle along the flight path, i.e. one half of the aperture's diameter. For a larger source, $b$ should perhaps an average between the diameter of the emitting source and the diameter of the aperture.

\section{A THERMAL STATE}

\section{A. Fully Normal Ordered Term in the Low Temperature Limit}

Now we turn to the case of a thermal state, so the fluctuations of the expansion have their origin in thermal fluctuations of the scalar field stress tensor. In this subsection, we consider the limit of a localized ray in the sense discussed above. The limit in which $a$ and $b$ are small is equivalent to a low temperature limit because the thermal wavelength is large compared to these scales. In this limit, we need to consider only the effect of the fully normal ordered term, so we must calculate the right-hand side of Eq. (20). There is a useful identity which can be used for this purpose:

$$
\left\langle: \phi_{1} \phi_{2} \phi_{3} \phi_{4}:\right\rangle=\left\langle: \phi_{1} \phi_{2}:\right\rangle\left\langle: \phi_{3} \phi_{4}:\right\rangle+\left\langle: \phi_{1} \phi_{3}:\right\rangle\left\langle: \phi_{2} \phi_{4}:\right\rangle+\left\langle: \phi_{1} \phi_{4}:\right\rangle\left\langle: \phi_{2} \phi_{3}:\right\rangle
$$


where the expectation values are taken in a thermal state. One proof of this identity is based upon the Hawking effect [20]. First, one may use Wick's theorem to prove Eq. (52) for a generalized vacuum state [8]. This is any state $|\psi\rangle$ for which there exists a decomposition of the field operators into positive and negative frequency parts,

$$
\phi=\phi^{+}+\phi^{-}
$$

such that $\phi^{+}|\psi\rangle=\langle\psi| \phi^{-}=0$. However, the formation of a black hole by gravitational collapse transforms the in-vacuum state into a thermal state at late times [20]. As a consequence, Eq. (52) must also be valid for thermal states.

It is also possible to give a more direct proof of this identity. Recall that the thermal average of an operator $A$ can be expressed as

$$
\langle A\rangle_{\beta}=\frac{\operatorname{Tr}(\rho A)}{\operatorname{Tr}(\rho)}
$$

where $\rho$ is the density operator. Let us now express each of the $\phi_{i}$ operators as a sum of positive and negative frequency components, as in Eq. (53). Recall that normal ordering means positive frequency terms are to the right of all negative frequency terms. Because $\rho$ has a Fock space representation as a sum of terms of the form $|n\rangle\langle n|$, where $|n\rangle$ is a number eigenstate, only terms with equal numbers of positive frequency and negative frequency factors can have nonzero expectation values. Thus, we can write

$$
\begin{aligned}
\left\langle: \phi_{1} \phi_{2} \phi_{3} \phi_{4}:\right\rangle & =\left\langle\phi_{1}^{-} \phi_{2}^{-} \phi_{3}^{+} \phi_{4}^{+}\right\rangle+\left\langle\phi_{1}^{-} \phi_{3}^{-} \phi_{2}^{+} \phi_{4}^{+}\right\rangle+\left\langle\phi_{1}^{-} \phi_{4}^{-} \phi_{2}^{+} \phi_{3}^{+}\right\rangle \\
& +\left\langle\phi_{2}^{-} \phi_{3}^{-} \phi_{1}^{+} \phi_{4}^{+}\right\rangle+\left\langle\phi_{2}^{-} \phi_{4}^{-} \phi_{1}^{+} \phi_{3}^{+}\right\rangle+\left\langle\phi_{3}^{-} \phi_{4}^{-} \phi_{1}^{+} \phi_{2}^{+}\right\rangle .
\end{aligned}
$$

Next we can use the fact that in a thermal state

$$
\left\langle a_{i}^{\dagger} a_{j}^{\dagger} a_{k} a_{l}\right\rangle=\left\langle a_{i}^{\dagger} a_{l}\right\rangle\left\langle a_{j}^{\dagger} a_{k}\right\rangle+\left\langle a_{i}^{\dagger} a_{k}\right\rangle\left\langle a_{j}^{\dagger} a_{l}\right\rangle
$$

to write

$$
\left\langle\phi_{1}^{-} \phi_{2}^{-} \phi_{3}^{+} \phi_{4}^{+}\right\rangle=\left\langle\phi_{1}^{-} \phi_{4}^{+}\right\rangle\left\langle\phi_{2}^{-} \phi_{3}^{+}\right\rangle+\left\langle\phi_{1}^{-} \phi_{3}^{+}\right\rangle\left\langle\phi_{2}^{-} \phi_{4}^{+}\right\rangle,
$$

and similar relations. We can now express $\left\langle: \phi_{1} \phi_{2} \phi_{3} \phi_{4}:\right\rangle$ as a sum of products of the form of that on the right-hand side of Eq. (157). By use of the fact that for a thermal state

$$
\left\langle: \phi_{1} \phi_{2}:\right\rangle=\left\langle\phi_{1}^{-} \phi_{2}^{+}\right\rangle+\left\langle\phi_{2}^{-} \phi_{1}^{+}\right\rangle
$$

this sum can be rewritten to complete the proof of Eq. (52).

By use of this identity, the fully normal ordered term, Eq. (20), can be expressed as

$$
C^{\mu \nu \alpha \gamma}\left(x, x^{\prime}\right)_{N O}=64 \pi^{2}\left(\partial^{\mu} \partial^{\prime \alpha} D_{\beta} \partial^{\nu} \partial^{\prime \gamma} D_{\beta}+\partial^{\nu} \partial^{\prime \alpha} D_{\beta} \partial^{\mu} \partial^{\prime \gamma} D_{\beta}\right)
$$

where

$$
D_{\beta}=\left\langle: \phi(x) \phi\left(x^{\prime}\right):\right\rangle_{\beta}=\sum_{n=-\infty}^{\infty} \frac{1}{4 \pi^{2}\left[|\Delta \mathbf{x}|^{2}-(\Delta t+i n \beta)^{2}\right]},
$$

is the renormalized thermal two-point function. Here $\beta=1 / T$, where $T$ is the temperature, $\Delta \mathbf{x}=\mathbf{x}-\mathbf{x}^{\prime}, \Delta t=t-t^{\prime}$, and the prime on the summation denotes that the $n=0$ term is omitted. 
Recall that here we are discussing a limit of localized rays. Let the unperturbed path of the bundle of geodesics be in the $+x$-direction, so that $\Delta x=\Delta t$ and $\Delta y=$ $\Delta z=0$. We can take the coordinate time in a given frame of reference to be the affine parameter, so that $\lambda=t, \lambda^{\prime}=t^{\prime}$ and $k^{\mu}=(1,1,0,0)$ in Eq. (15). After some calculation, the integrand may be expressed as

$$
C^{\mu \nu \alpha \gamma} v_{\mu} v_{\nu} v_{\alpha} v_{\gamma}=C_{N O}^{\mu \nu \alpha \gamma} v_{\mu} v_{\nu} v_{\alpha} v_{\gamma}=\frac{2^{11}}{\pi^{2} \beta^{8}} f(k)
$$

where

$$
\begin{aligned}
f(k) & =\left[\sum_{n=1}^{\infty} \frac{\left(n^{2}-3 k^{2}\right)}{\left(n^{2}+k^{2}\right)^{3}}\right]^{2} \\
& =\frac{1}{4 k^{8}}\left[\pi^{3} k^{3} \operatorname{coth}(\pi k) \operatorname{csch}^{2}(\pi k)+\pi^{2} k^{2} \operatorname{csch}^{2}(\pi k)+\pi k \operatorname{coth}(\pi k)-3\right]^{2}(62)
\end{aligned}
$$

and $k=2 \Delta t / \beta$. Now we can write the variance of the expansion as

$$
\left\langle(\Delta \theta)^{2}\right\rangle=\int_{0}^{s} d t \int_{0}^{s} d t^{\prime} C_{N O}^{\mu \nu \alpha \gamma} k_{\mu} k_{\nu} k_{\alpha} k_{\gamma}=\frac{2^{10}}{\pi^{2} \beta^{6}} \int_{0}^{\kappa} d k(\kappa-k) f(k) .
$$

Here $s$ is the separation of the source and detector (the flight time), $\kappa=2 s / \beta$, and we have used Eq. (33). The function $f(k)$ is finite at $k=0, f(0)=\pi^{8} / 8100$ and vanishes rapidly for large $k$ :

$$
f(k) \sim \frac{\pi^{2}}{4 k^{6}}, \quad k \rightarrow \infty
$$

As a consequence, both $\int_{0}^{\infty} d k f(k)$ and $\int_{0}^{\infty} d k k f(k)$ are finite. In this case, the large $\kappa$ limit of Eq. (63) is

$$
\left\langle(\Delta \theta)^{2}\right\rangle=\frac{2^{10} c_{0}}{\pi^{2} \beta^{6}} \kappa
$$

where

$$
c_{0}=\int_{0}^{\infty} d k f(k) \approx 0.3468 .
$$

We can now write the root-mean-squared fluctuation in the expansion as

$$
\Delta \theta_{r m s}=\frac{128 \sqrt{c_{0}}}{\pi} \ell_{P}^{2} \sqrt{s T^{7}}
$$

The fractional luminosity fluctuations of the image of a point source viewed at a distance $s$ through a bath of scalar radiation at temperature $T$ becomes, for localized rays,

$$
\left(\frac{\Delta L}{L}\right)_{r m s}=\frac{64 \sqrt{c_{0}}}{\sqrt{3} \pi} \ell_{P}^{2} \sqrt{s^{3} T^{7}}
$$


Equation (68) can be expressed as

$$
\left(\frac{\Delta L}{L}\right)_{r m s}=0.02\left(\frac{s}{10^{28} \mathrm{~cm}}\right)^{\frac{3}{2}}\left(\frac{T}{10^{6} K}\right)^{\frac{7}{2}}=10^{-3}\left(\frac{s}{10^{6} \mathrm{~km}}\right)^{\frac{3}{2}}\left(\frac{T}{1 \mathrm{GeV}}\right)^{\frac{7}{2}} .
$$

Thus a source seen at a cosmological distance, $10^{28} \mathrm{~cm} \approx 3 \mathrm{Gpc}$ through a thermal bath at a temperature of $10^{6} \mathrm{~K}$ could in principle show large luminosity fluctuations if one could select rays localized on a scale less than the thermal wavelength $\beta$. Of course, there cannot be such a bath in our present universe, as its energy density would have long dominated the expansion of the universe. Note that $10^{6} \mathrm{~km}$ is the Schwarzschild radius of a black hole with a mass of $3 \times 10^{5} M_{\odot}$, in the mass range of the supermassive black holes which may be in the centers of many galaxies, and possible linked to the phenomenon of gamma ray bursts. In at least some cases [22], these bursts have produced gamma rays with energies well in excess of $1 \mathrm{GeV}$. Although the details of the size and nature of the emitting regions is unclear, this raises the possibility that within the burst sources, there are quantum metric fluctuations large enough to achieve $\Delta L / L \approx 1$ for a bundle of rays localized on a scale small compared to thermal wavelength $\beta$. Any realistic observation is likely to average over a much larger scale, so we now turn to the opposite limit.

\section{B. High Temperature Limit}

In the limit in which the averaging scales $a$ and $b$ are large compared to the thermal wavelength, we need to adopt a different approach. Now the effect of the averaging cannot be ignored, even in the fully normal-ordered term. It is easiest to include the effects of the vacuum, cross, and fully normal-ordered terms all together by using the full thermal Green's function,

$$
D_{F \beta}=\left\langle: \phi(x) \phi\left(x^{\prime}\right):\right\rangle_{\beta}=\sum_{n=-\infty}^{\infty} \frac{1}{4 \pi^{2}\left[|\Delta \mathbf{x}|^{2}-(\Delta t+i n \beta)^{2}\right]} .
$$

If we impose the same type of averaging as used in Sect. IIIC, then we can write

$$
\begin{aligned}
& \left\langle(\Delta \theta)^{2}\right\rangle= \\
& \frac{512}{\pi^{2}}\left[\int_{0}^{v_{0}} d v\left(v_{0}-v\right)+\int_{-v_{0}}^{0} d v\left(v_{0}+v\right)\right] \int_{-\infty}^{\infty} d u g_{L}(\Delta u, a) \int d^{2} x g(r)\left(\partial_{v}^{2} D_{F \beta}\right)^{2} .
\end{aligned}
$$

In the high temperature (small $\beta$ ) limit, we can replace the sum on $n$ in $D_{F \beta}$ by an integral. Let $\xi=\beta n$, and let

$$
\sum_{n=-\infty}^{\infty} \rightarrow \frac{1}{\beta} \int_{-\infty}^{\infty} d \xi
$$

Then we can write

$$
\partial_{v}^{2} D_{F \beta}=\frac{16}{\pi^{2} \beta} \int_{-\infty}^{\infty} d \xi \frac{(i \xi+u)^{2}}{\left[(v-u)^{2}+4 r^{2}-(2 i \xi+v+u)^{2}\right]^{3}}=-\frac{1}{2 \pi \beta} \frac{2 r^{2}-(v-u)^{2}}{\left[(v-u)^{2}+4 r^{2}\right]^{\frac{5}{2}}} .
$$


This leads to

$$
\begin{aligned}
& \left\langle(\Delta \theta)^{2}\right\rangle=\frac{32}{\beta^{2}} \int_{-\infty}^{\infty} d u g_{L}(\Delta u, a) \int d^{2} x g(r) \times \\
& {\left[\int_{0}^{v_{0}} d v\left(v_{0}-v\right)+\int_{-v_{0}}^{0} d v\left(v_{0}+v\right)\right] \frac{\left[2 r^{2}-(v-u)^{2}\right]^{2}}{\left[(v-u)^{2}+4 r^{2}\right]^{5}} .}
\end{aligned}
$$

In the limit that $v_{0}$ is large compared to either $a$ or $b$, we can write this as

$$
\left\langle(\Delta \theta)^{2}\right\rangle=\frac{32 v_{0}}{\beta^{2}} \int_{-\infty}^{\infty} d u g_{L}(\Delta u, a) \int d^{2} x g(r) \int_{-\infty}^{\infty} d v \frac{\left(2 r^{2}-v^{2}\right)^{2}}{\left(v^{2}+4 r^{2}\right)^{5}}=\frac{27 \pi v_{0}}{512 \beta^{2}}\left\langle\frac{1}{r^{5}}\right\rangle .
$$

Note that in this limit, the integrand of the $v$-integration becomes independent of $u$, and hence the result is independent of $a$.

The final expression involves the transverse average of a singular function, $1 / r^{5}$. However, unlike the singular functions encountered earlier in the vacuum and cross terms, this is simply an artifact of our approximations. The dominant contribution here is from the fully-normal ordered term, and should be finite. The apparent singularity at $r=0$ arises because our approximation of replacing the sum by an integral assumes that $\beta$ is small compared to all other length scales including $r$. An exact calculation of the fully-normal ordered contribution to $\left\langle(\Delta \theta)^{2}\right\rangle$ would, in the high temperature limit, replace Eq. (75) by

$$
\left\langle(\Delta \theta)^{2}\right\rangle=\frac{27 \pi v_{0}}{512 \beta^{2}}\langle f(r)\rangle,
$$

where $f(r)$ is a function which falls as $r^{-5}$ for $r \gtrsim \beta$, but approaches a constant for $r \lesssim \beta$. We can estimate $\langle f(r)\rangle$ in the high temperature limit by simply cutting off the integration on $r$ at a lower limit of order $\beta$. Thus,

$$
\langle f(r)\rangle \approx \frac{2}{b^{2}} \int_{\beta}^{\infty} \frac{d r}{r^{4}} \mathrm{e}^{-r^{2} / b^{2}} \approx \frac{2}{3 b^{2} \beta^{3}} .
$$

This leads to an estimate for $\left\langle(\Delta \theta)^{2}\right\rangle$ of

$$
\left\langle(\Delta \theta)^{2}\right\rangle=\frac{K^{2}}{b^{2} \beta^{5}} s
$$

where $K$ is a constant of order one.

The corresponding fractional luminosity fluctuation is

$$
\left(\frac{\Delta L}{L}\right)_{r m s}=\frac{K s^{\frac{3}{2}}}{b \beta^{\frac{5}{2}}} \ell_{P}^{2} .
$$

Note that as compared to Eq. (68), the effect is now suppressed by a factor of $\beta / b$. This is essentially the effect of classical averaging of the luminosity fluctuations; when one averages over a larger source, the fluctuations are suppressed. In Eq. (79), we might take $b$ to be either of the order of the size of the source, or of the average of the source size and the observer's aperture size. In either case, the luminosity fluctuations will be strongly suppressed compared to the estimates given in Eq. (69). 


\section{DISCUSSION}

In this paper, we have treated the effects of the fluctuations of a massless, minimally coupled scalar field stress tensor on a flat background. In particular, we used the fluctuations in expansion of a congruence of null rays as an operational measure of the effects of stress tensor fluctuations on the spacetime geometry. Although the cross and vacuum terms are potentially singular parts of the stress tensor correlation function, we argued that their contributions can be rendered finite by an integration by parts procedure. In the limit of localized rays, they actually yield a zero contribution to the variance of the expansion. This is in contrast to other situations [4, 5], where the cross term often yields the dominant contribution. We calculated an explicit formulas for the fluctuations in the expansion produced by a thermal bath. This in turn led to a results, Eqs. (68) and (79), for the fractional luminosity fluctuations of the image of an object seen through a fluctuating spacetime filled with a thermal bath. Although these effects are usually small, there are conceivable astrophysical situations where $\Delta L / L$ would become large if the rays could be sufficiently localized.

The effects of the fully-normal ordered term are unambiguous, but those of the vacuum and cross terms contain singular parts which need to be defined by a spacetime averaging over a bundle of rays. This may be done by the integration by parts procedure discussed in Appendix C. This procedure involves some undetermined constants, some of which can be eliminated by a scaling argument. Unfortunately, the overall constant which determines the magnitude of the vacuum contribution is not fixed. Further work is needed to determine this constant uniquely. As noted above, one topic for future research will be the use of covariant averaging.

Note that Eq. (67) describes the effects of the Ricci tensor fluctuations, not the expansion due to any mean Ricci curvature. Our treatment has assumed a nearly flat background spacetime, More generally, the non-zero mean value of the stress tensor will lead to mean Ricci curvature and hence classical focusing.

Recall that we assumed that the $\theta^{2}$ term in the Raychaudhuri equation, Eq. (12), can be neglected. As $\left\langle(\Delta \theta)^{2}\right\rangle$ grows, this assumption will eventually break down. However, in many cases $\left\langle(\Delta \theta)^{2}\right\rangle$ remains small compared to the Ricci tensor term for a very long time. We can give an explicit estimate of this time for the case of the thermal bath. In this case, the root-mean-square fluctuations in the Ricci tensor term are of order $\left|R_{\mu \nu} v^{\mu} v^{\nu}\right|_{r m s} \approx \ell_{P}^{2} T^{4}$. Thus $\left\langle(\Delta \theta)^{2}\right\rangle \ll\left|R_{\mu \nu} v^{\mu} v^{\nu}\right|_{r m s}$ so long as

$$
s \ll \frac{\beta^{3}}{\ell_{P}^{2}} .
$$

For temperatures less than about $1 \mathrm{GeV}$, this condition will be satisfied for any $s$ less than the size of the observable universe. We also assumed that the shear and vorticity terms in the Raychaudhuri equation can be ignored. So long as the bundle of geodesics is hypersurface orthogonal, the vorticity remains zero in all classical spacetimes, so it is reasonable to expect that small quantum geometry fluctuations will not generate any vorticity. The shear can grow in response to non-zero Weyl curvature. Thus if the stress tensor fluctuations cause the Weyl tensor to fluctuate, through the excitation of gravitational wave modes, then eventually $\left\langle\sigma_{\mu \nu} \sigma^{\mu \nu}\right\rangle$ could become important. However, it seems reasonable to speculate that it will not grow any more rapidly than does $\left\langle(\Delta \theta)^{2}\right\rangle$. 
In summary, we have illustrated how expansion fluctuations provide a concrete measure of the effects of passive quantum geometry fluctuations.

\section{Acknowledgments}

We would like to thank Arvind Borde, Ken Olum, Tom Roman, Richard Woodard, and Chun-Hsien Wu for useful discussions. This work was supported in part by the National Science Foundation under Grant PHY-0244898.

\section{APPENDIX A}

In this Appendix, we will illustrate the geometric meaning of the expansion $\theta$ in an explicit example [23], and then use this example to infer a relation between fluctuations of the expansion and the angular blurring of an image. First consider a point source in flat spacetime which is emitting null rays, whose tangent vector field is $v^{\mu}=d x^{\mu} / d \lambda$. Choose a coordinate system in which the origin of spatial coordinates is at the location of the point source, and take the affine parameter to be coordinate time in this frame: $\lambda=t$. Then

$$
v^{\mu}=(1, \mathbf{v}),
$$

where $\mathbf{v}$ is a unit 3 -vector, which is also the unit position vector in this coordinate system

$$
\mathbf{v}=\hat{\mathbf{x}}=\frac{\mathbf{x}}{|\mathbf{x}|}
$$

The expansion is defined as the trace of the tensor $\nabla^{\nu} v^{\mu}$ in the subspace orthogonal to $v^{\mu}$ :

$$
\theta=\nabla^{\nu} v^{\mu}\left(g_{\mu \nu}+v_{\mu} v_{\nu}\right)
$$

In our case, the tensor $\nabla^{\nu} v^{\mu}$ has only spatial components

$$
\nabla^{j} v^{i}=\partial^{j} v^{i}=\frac{\delta^{i j}|\mathbf{x}|^{2}-x^{i} x^{j}}{|\mathbf{x}|^{3}} .
$$

Now $\theta$ can be found to be

$$
\theta=\partial^{j} v^{i}\left(\delta_{i j}+v_{i} v_{j}\right)=\frac{2}{s}
$$

where $s=|\mathbf{x}|$ is the distance from the source. If we repeat this calculation for the case of timelike geodesics, using the 4 -velocity $u^{\mu}$ as the tangent vector, the result is

$$
\theta=\frac{2 \gamma v}{s}
$$



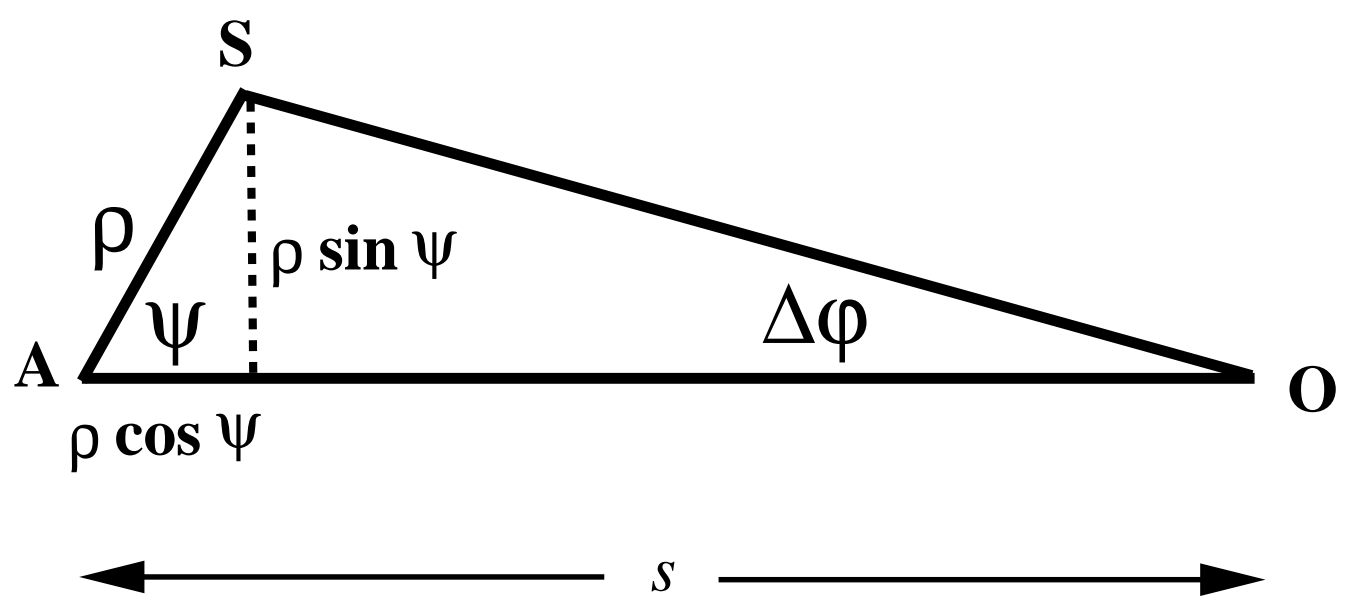

FIG. 1: A source $S$ at a mean distance $s$ from an observer $O$ is displaced in direction $\psi$ by a distance $\rho$ from its average position $A$. The effect is to shift the apparent position of the source by an angle $\Delta \varphi$.

where $\gamma=1 / \sqrt{1-v^{2}}$. In both cases, $\theta \propto 1 / s$, so as the distance from the source increases and the rays become more nearly parallel, the expansion decreases.

Now let us relate the fluctuations of $\theta$ to fluctuations in the apparent angular position of the source. In our case, the $\theta$-fluctuations are due to fluctuations in the spacetime geometry between the source and the observer. However, one could achieve the same effect with a source whose position fluctuates in a fixed Minkowski spacetime. Consider the situation illustrated in Fig. 11, where the source is displaced from its mean position by a distance $\rho$ in a direction given by the angle $\psi$. For an observer at a mean distance of $s$, the expansion changes by

$$
\Delta \theta=-\frac{2}{s^{2}} \Delta s=\frac{2}{s^{2}} \rho \cos \psi,
$$

and the angular position of the source changes by approximately

$$
\Delta \varphi=\frac{\rho \sin \psi}{s},
$$

provided that $\rho \ll s$. We now assume that $\rho$ and $\psi$ fluctuate independently of one another and that all values of $\psi$ are equally probable. Then

$$
\left\langle\rho^{2} \sin ^{2} \psi\right\rangle=\left\langle\rho^{2}\right\rangle\left\langle\sin ^{2} \psi\right\rangle=\frac{1}{2}\left\langle\rho^{2}\right\rangle
$$

and

$$
\left\langle\rho^{2} \cos ^{2} \psi\right\rangle=\left\langle\rho^{2}\right\rangle\left\langle\cos ^{2} \psi\right\rangle=\frac{1}{2}\left\langle\rho^{2}\right\rangle .
$$


This would happen, for example, if $\rho$ does not fluctuate and $\psi$ fluctuates randomly. This allow us to relate the expansion fluctuations to the angular position fluctuations:

$$
\left\langle(\Delta \varphi)^{2}\right\rangle=\frac{1}{4} s^{2}\left\langle(\Delta \theta)^{2}\right\rangle
$$

Thus the effect of $\theta$-fluctuations is to blur the angular resolution of the image of the source by an amount

$$
\Delta \varphi_{\mathrm{rms}}=\frac{1}{2} s \Delta \theta_{\mathrm{rms}}
$$

Strictly, we have treated only a source in flat spacetime whose position fluctuates. However, we offer this as a heuristic estimate of the magnitude of angular blurring expected in a fluctuating spacetime.

\section{APPENDIX B}

In this appendix, we show how the stochastic behavior of the expansion leads to small deviations in the density of geodesics. In the case of light rays this translates into luminosity fluctuations. The connection between luminosity fluctuations and expansion fluctuations can be seen as follows. Let us write the expansion as the sum of a classical (deterministic) component $\theta_{c}$ and a stochastic component $\hat{\theta}$ :

$$
\theta(t)=\theta_{c}(t)+\hat{\theta}(t)
$$

Here $t$ is an affine parameter along the bundle of geodesics. The stochastic component can expresse as

$$
\hat{\theta}=\frac{d \log \left(A / A_{c}\right)}{d t}
$$

where $\hat{A}$ is the cross sectional area of a bundle. Now we assume $\hat{\theta}$ is a small stochastic perturbation. This means

$$
A=\hat{A}+A_{c} \approx A_{c}
$$

where $\hat{A}$ is the stochastic variation in the area (zero at $t=0$ ). Using the Taylor expansion of $\log (1+\mathrm{x})$ for small $\mathrm{x}$, we find

$$
\begin{aligned}
\hat{\theta} & \approx \frac{d\left(\hat{A} / A_{c}\right)}{d t}, \\
\int_{t_{0}}^{t} d t^{\prime} \theta\left(t^{\prime}\right) & \approx \frac{\hat{A}}{A_{c}}=\frac{\Delta \rho}{\rho},
\end{aligned}
$$

where $\rho$ is the density of geodesics. Since the number of geodesics, $n$, is conserved (total energy flow), while the transverse area $A$ is distorted (density fluctuations),

$$
\text { Intensity or Luminosity }=\frac{n}{A} \text {. }
$$


The fractional luminosity fluctuation is therefore given by

$$
\frac{\Delta L}{L}=\frac{\Delta \rho}{\rho} \approx \int_{t_{0}}^{t} d t^{\prime} \theta\left(t^{\prime}\right) .
$$

Now the variance of the fractional luminosity fluctuations can be computed as

$$
\left\langle\left(\frac{\Delta L}{L}\right)^{2}\right\rangle=\int_{0}^{s} \int_{0}^{s} d t^{\prime} d t^{\prime \prime}\left\langle\theta\left(t^{\prime}\right) \theta\left(t^{\prime \prime}\right)\right\rangle .
$$

For large flight times (where $s$ in the upper bound of the integral is much greater than the correlation length scale), there is a simple connection between the variance of the fractional luminosity fluctuations and the variance of the expansion:

$$
\left\langle\left(\frac{\Delta L}{L}\right)^{2}\right\rangle \propto s^{2}\left\langle\theta^{2}(s)\right\rangle .
$$

Let us show this for a typical positive-definite correlation function, $C_{1}(\Delta t)$. We have

$$
\left\langle\theta(t) \theta\left(t^{\prime}\right)\right\rangle=\int_{0}^{t} d t_{1} \int_{0}^{t^{\prime}} d t_{2} C_{1}(|\Delta t|) .
$$

The function $C_{1}$ is symmetric in its arguments, so just consider $t<t^{\prime}$, then symmetrize later. The first integration over $d t_{2}$ produces the area under the Ricci correlation curve, $c_{o}$. (We will be considering large flight times, so the edge effect at the origin is neglected.) After the second integration, and symmetrizing, we have the expression for the expansion correlation function:

$$
\begin{aligned}
\left\langle\theta(t) \theta\left(t^{\prime}\right)\right\rangle & =c_{0} t, \quad t^{\prime}>t, \\
& =c_{0} t^{\prime}, \quad t>t^{\prime} .
\end{aligned}
$$

Now we can calculate the fractional luminosity fluctuations:

$$
\begin{aligned}
\left\langle\left(\frac{\Delta L}{L}\right)^{2}\right\rangle & =\int_{0}^{s} \int_{0}^{s} d t d t^{\prime}\left\langle\theta(t) \theta\left(t^{\prime}\right)\right\rangle \\
& =2 \int_{0}^{s} d t \int_{0}^{s} d t^{\prime}\left\langle\theta(t) \theta\left(t^{\prime}\right)\right\rangle, \quad t>t^{\prime}, \\
& =2 c_{0} \int_{0}^{s} d t \int_{0}^{t} d t^{\prime} t^{\prime} \\
& =c_{0} \int_{0}^{s} d t t^{2} \\
& =\frac{1}{3} c_{0} s^{3} \\
& =\frac{1}{3} s^{2}\left\langle\theta(s)^{2}\right\rangle .
\end{aligned}
$$

Explicit calculations show that Eq.(B9) also holds for the vacuum correlation function encountered in this paper. In the case of the vacuum term, direct calculation reveals

$$
\left\langle\left(\frac{\Delta L}{L}\right)^{2}\right\rangle_{v a c}=\frac{1}{4} s^{2}\left\langle\theta(s)^{2}\right\rangle_{v a c} .
$$




\section{APPENDIX C}

In this Appendix, we will discuss two methods for defining quantities such as $\left\langle 1 / r^{8}\right\rangle$. The first approach involves an integration by parts. Let us first illustrate this approach in one space dimension with the quantity

$$
\rho_{n}^{(1)}=\left\langle x^{n}\right\rangle=\int_{-\infty}^{\infty} d x x^{n} g_{1}(x)
$$

where $g_{1}(x)=\mathrm{e}^{-x^{2} / b^{2}} /(\sqrt{\pi} b)$. If $n>-1$, then the above integral is absolutely convergent and there is no ambiguity in the definition of $\rho_{n}^{(1)}$. If, however, $n<-1$, then the integral in Eq. (C1) diverges at $x=0$. In the case that $n$ is a negative integer, let $m=-n$ and write

$$
x^{n}=\frac{1}{x^{m}}=\frac{(-1)^{m-1}}{2(m-1) !} \frac{d^{m}}{d x^{m}}\left[\ln \left(\frac{x^{2}}{x_{0}^{2}}\right)+P_{m-1}(x)\right] \text {, }
$$

where $x_{0}$ is a constant and $P_{m-1}(x)$ is an arbitrary $(m-1)$-th degree polynomial in $x$. We can now insert this identity into Eq. (C1) and integrate by parts to define

$$
\rho_{n}^{(1)}=\left\langle\frac{1}{x^{m}}\right\rangle=-\frac{1}{2(m-1) !} \int_{-\infty}^{\infty} d x\left[\ln \left(\frac{x^{2}}{x_{0}^{2}}\right)+P_{m-1}(x)\right] \frac{d^{m}}{d x^{m}} g_{1}(x) \text {. }
$$

The resulting integrals are not only convergent, but are also independent of the choices of $x_{0}$ and the coefficients of $P_{m-1}(x)$. (These are not really independent, as a shift in $x_{0}$ shifts the zeroth order term in $P_{m-1}$.) Furthermore, the results are always of order $b^{-m}$. For example,

$$
\begin{gathered}
\left\langle 1 / x^{2}\right\rangle=-2 / b^{2}, \\
\left\langle 1 / x^{4}\right\rangle=4 /\left(3 b^{4}\right), \\
\left\langle 1 / x^{6}\right\rangle=-8 /\left(15 b^{6}\right),
\end{gathered}
$$

and

$$
\left\langle 1 / x^{8}\right\rangle=16 /\left(105 b^{8}\right)
$$

Note that if we were to allow the polynomial $P_{m-1}$ to be a function of $|x|$, rather than $x$, then the final result will depend upon the coefficients of the odd powers of $|x|$. For example, let

$$
P_{1}=P_{1}(|x|)=c_{0}+c_{1}|x| \text {. }
$$

Then we find

$$
\rho_{-2}^{(1)}=-\frac{2}{b^{2}}-\frac{c_{1}}{\sqrt{\pi} b}
$$

In effect, what has happened here is the addition of a term proportional to $\delta(x)$ into the representation of $1 / x^{2}$, which has caused $\rho_{-2}^{(1)}$ to acquire a term proportional to $g_{1}(0)$ with an arbitrary coefficient. 
The second approach to defining the $\rho_{n}^{(1)}$ involves a recurrence relation. Note that if we let $\alpha=1 / b^{2}$, then we can write

$$
\frac{\partial g_{1}}{\partial \alpha}=\left(\frac{1}{2 \alpha}-x^{2}\right) g_{1}
$$

This allows us to derive the recurrence relation

$$
\rho_{n+2}^{(1)}=-\sqrt{\alpha} \frac{d}{d \alpha}\left(\frac{\rho_{n}^{(1)}}{\sqrt{\alpha}}\right) .
$$

We can start from $\rho_{0}^{(1)}=1$ and uniquely generate all $\rho_{n}^{(1)}$, where $n$ is a positive even integer from Eq. (C11). We can also integrate this relation to define $\rho_{n}^{(1)}$ for negative even values of $n$, but the result will contain undetermined constants of integration which are equivalent to the arbitrary coefficients in $P_{m-1}(|x|)$.

Now we turn to two space dimensions and examine the quantities

$$
\rho_{n}^{(2)}=\left\langle r^{n}\right\rangle=\int d^{2} x r^{n} g(r)=2 \pi \int_{0}^{\infty} d r r^{n+1} g(r)
$$

where the sampling function is now $g(r)=\mathrm{e}^{-r^{2} / b^{2}} /\left(\pi b^{2}\right)$. Here the integrals converge if $n>-2$, but are divergent for $n \leq-2$. In the latter case, we can still use integration by parts to define $\rho_{n}^{(2)}$. Consider first the case where $n=-2 \ell$ is a negative, even integer, and write

$$
\frac{1}{r^{2 \ell}}=\left(\nabla^{2}\right)^{\ell}\left[a_{\ell} \ln ^{2}\left(\frac{r^{2}}{r_{0}^{2}}\right)+f_{\ell}(r)\right]
$$

where

$$
\nabla^{2}=\frac{1}{r} \frac{d}{d r}\left(r \frac{d}{d r}\right)
$$

is the Laplacian operator and $f_{m}(r)$ is any function which satisfies

$$
\left(\nabla^{2}\right)^{\ell} f_{\ell}(r)=0
$$

for $r \neq 0$. If we insert Eq. (C13) into Eq. (C12), the Laplacian operators may be successively moved to act on $g(r)$ by repeated use of Green's theorem:

$$
\int\left(F \nabla^{2} G-G \nabla^{2} F\right) d V=\oint(F \nabla G-G \nabla F) d a .
$$

Here the region of integration is a disk of radius $R$. In the limit that $R \rightarrow \infty$, the surface term vanishes because $g(r)$ and its derivatives vanish rapidly. The result is

$$
\rho_{-2 \ell}^{(2)}=\left\langle\frac{1}{r^{2 \ell}}\right\rangle=2 \pi \int_{0}^{\infty} d r r\left[a_{\ell} \ln ^{2}\left(\frac{r^{2}}{r_{0}^{2}}\right)+f_{\ell}(r)\right]\left(\nabla^{2}\right)^{\ell} g(r) .
$$

This integral is convergent, but depends upon various arbitrary constants. 
This is best illustrated with some specific examples. First let $\ell=1$, in which case we find $a_{1}=1 / 8$ and

$$
f_{1}=c_{0} \ln \left(\frac{r}{c_{1}}\right)
$$

This leads to the result

$$
\left\langle\frac{1}{r^{2}}\right\rangle=-\frac{1}{b^{2}}\left[2 \ln \left(\frac{r_{0}}{b}\right)+\gamma+c_{0}\right]
$$

where $\gamma$ is Euler's constant. There is really one arbitrary constant in this result, as $c_{0}$ may be absorbed into a redefinition of the constant $r_{0}$. We can understand the appearance of $c_{0}$ in Eq.(C19) from the fact that

$$
\nabla^{2} f_{1}(r)=-2 \pi c_{0} \delta(\mathbf{x})
$$

in two dimensions. Similar results arise for larger values of $\ell$, but with more constants, For $\ell=2$, where $a_{2}=1 / 32$, we can solve $\nabla^{2} f_{2}=f_{1}$ to find

$$
f_{2}=\frac{1}{4} c_{0} r^{2}\left[\ln \left(\frac{r}{c_{1}}\right)-1\right]+c_{2} \ln \left(\frac{r}{c_{3}}\right)
$$

and

$$
\left\langle\frac{1}{r^{4}}\right\rangle=\frac{1}{b^{4}}\left[2 \ln \left(\frac{r_{0}}{b}\right)+\gamma+1-8 c_{2}\right]+\frac{2 c_{0}}{b^{2}} .
$$

For $\ell=3, a_{3}=1 / 512$ and

$$
f_{3}=\frac{1}{128} c_{0} r^{4}\left[2 \ln \left(\frac{r}{c_{1}}\right)-3\right]+\frac{1}{4} c_{2} r^{2}\left[\ln \left(\frac{r}{c_{3}}\right)-1\right]+c_{4} \ln \left(\frac{r}{c_{5}}\right),
$$

which yields

$$
\left\langle\frac{1}{r^{6}}\right\rangle=-\frac{1}{4 b^{6}}\left[4 \ln \left(\frac{r_{0}}{b}\right)+2 \gamma+3-64 c_{4}\right]-\frac{8 c_{2}}{b^{4}}+\frac{2 c_{0}}{b^{2}} .
$$

Finally for $\ell=4$, where $a_{4}=1 / 18432$,

$$
\begin{aligned}
f_{4} & =\frac{1}{13824} c_{0} r^{6}\left[6 \ln \left(\frac{r}{c_{1}}\right)-11\right]+\frac{1}{128} c_{2} r^{4}\left[2 \ln \left(\frac{r}{c_{1}}\right)-3\right] \\
& +\frac{1}{4} c_{4} r^{2}\left[\ln \left(\frac{r}{c_{3}}\right)-1\right]+c_{6} \ln \left(\frac{r}{c_{7}}\right)
\end{aligned}
$$

and

$$
\left\langle\frac{1}{r^{8}}\right\rangle=\frac{1}{36 b^{8}}\left[12 \ln \left(\frac{r_{0}}{b}\right)+6 \gamma+11\right]-\frac{768 c_{6}}{b^{8}}+\frac{64 c_{4}}{b^{6}}-\frac{8 c_{2}}{b^{4}}+\frac{2 c_{0}}{b^{2}} .
$$

Now let us consider the case where $n=-2 \ell-1$ is a negative odd integer and write

$$
\frac{1}{r^{2 \ell+1}}=\left(\nabla^{2}\right)^{\ell}\left[\frac{1}{[(2 \ell-1) ! !]^{2} r}+f_{\ell}(r)\right] \text {. }
$$


A procedure analogous to that in the case of even $n$ leads to

$$
\rho_{-2 \ell-1}^{(2)}=\left\langle\frac{1}{r^{2 \ell+1}}\right\rangle=2 \pi \int_{0}^{\infty} d r\left[\frac{1}{[(2 \ell-1) ! !]^{2}}+r f_{\ell}(r)\right]\left(\nabla^{2}\right)^{\ell} g(r) .
$$

The results for $\ell=1$ and $\ell=2$ are

$$
\left\langle\frac{1}{r^{3}}\right\rangle=-\frac{2 \sqrt{\pi}}{b^{3}}+\frac{2 c_{0}}{b^{2}}
$$

and

$$
\left\langle\frac{1}{r^{5}}\right\rangle=\frac{4 \sqrt{\pi}}{3 b^{5}}-\frac{8 c_{2}}{b^{4}}+\frac{2 c_{0}}{b^{2}} .
$$

We can also derive a recurrence relation for $\rho_{n}^{(2)}$. Note that

$$
\frac{\partial g}{\partial \alpha}=\left(\frac{1}{\alpha}-r^{2}\right) g .
$$

This leads to the relation

$$
\rho_{n+2}^{(2)}=-\alpha \frac{d}{d \alpha}\left(\frac{\rho_{n}^{(2)}}{\alpha}\right) .
$$

We may start from either $\rho_{0}^{(2)}=1$ or $\rho_{-1}^{(2)}=\sqrt{\pi} / b$ and integrate this recurrence relation to find $\rho_{n}^{(2)}$ for all negative integers $n$. The results agree with those of the integration by parts approach, with the constants $c_{i}$ arising as constants of integration.

Now we wish to address the difficult question of whether these constants can be determined by physical arguments. Let us first consider the one-dimensional case. So long as we require the polynomial $P_{m-1}$ to be a function of $x$, as opposed to $|x|$, then

$$
\frac{d^{m}}{d x^{m}} P_{m-1}=0
$$

and there is no ambiguity. The undetermined constants which appear if $P_{m-1}$ is a polynomial in $|x|$, or if we integrate the recurrence relation, all have dimensions of inverse powers of length. One way to resolve the ambiguity is to require that $\rho_{n}^{(1)}$ be proportional to $b^{n}$, which sets all of the constants of integration to zero. This is essentially a scaling requirement, that if $b \rightarrow 2 b$, for example, then $\rho_{n}^{(1)} \rightarrow 2^{n} \rho_{n}^{(1)}$. Another way to say this is that $\rho_{n}^{(1)}$ should depend only upon the sampling scale $b$ and not on any additional length scales.

For odd $n$, we can apply the same requirement to the two-dimensional case, and similarly require that

$$
\rho_{n}^{(2)} \propto b^{n} .
$$

In the case that $n$ is a negative odd integer, this uniquely defines $\rho_{n}^{(2)}$ by setting the constants $c_{1}=0$. For example, now

$$
\left\langle\frac{1}{r^{3}}\right\rangle=-\frac{2 \sqrt{\pi}}{b^{3}}
$$


and

$$
\left\langle\frac{1}{r^{5}}\right\rangle=\frac{4 \sqrt{\pi}}{3 b^{5}}
$$

For $n$ even and negative, there is still an undetermined multiplicative constant associated with a term logarithmic in $b$, so we will have

$$
\left\langle\frac{1}{r^{2 \ell}}\right\rangle=\frac{A_{2 \ell}}{b^{2 \ell}}
$$

where we allow $A_{2 \ell}$ to possess a possible logarithmic dependence on $b$.

[1] G. Barton, J. Phys. A 24, 991 (1991); 24, 5533 (1991).

[2] C. Eberlein, J. Phys. A 25, 3015 (1992); A 25, 3039 (1992).

[3] M.T. Jaekel and S. Reynaud, Quantum Opt. 4, 39 (1992); J. Phys. I France 2, 149 (1992); 3, 1 (1993); 3, 339 (1993).

[4] C.-H. Wu, Chung-I Kuo and L.H. Ford, Phys. Rev. A 65, 062102 (2002), quant-ph/0112056

[5] C.-H. Wu and L. H. Ford, Phys. Rev. D 64, 045010 (2001), quant-ph/0012144.

[6] L.H. Ford, Ann. Phys (NY) 144, 238 (1982).

[7] S. del Campo and L.H. Ford, Phys. Rev. D 38, 3657 (1988).

[8] Chung-I Kuo and L.H. Ford, Phys. Rev. D 47, 4510 (1993), gr-qc/9304008.

[9] N.G. Phillips and B.L. Hu, Phys. Rev. D 55, 6123 (1997), gr-qc/9611012.

[10] E. Calzetta and B.L. Hu, Phys. Rev. D 49, 6636 (1993), gr-qc/9312036 Phys. Rev. D 52, 6770 (1995), gr-qc//9505046.

[11] E. Calzetta, A. Campos, and E. Verdaguer, Phys. Rev. D 56, 2163 (1997), gr-qc/9704010.

[12] R. Martin and E. Verdaguer, Phys. Rev. D 60, 084008 (1999); gr-qc/9904021.

[13] B. L. Hu and K. Shiokawa, Phys. Rev. D 57, 3474 (1998); gr-qc/9708023.

[14] C.-H. Wu and L.H. Ford, Phys. Rev. D 60, 104013 (1999), gr-qc/9905012.

[15] C. Barrabes, V. Frolov, and R. Parentani, Phys. Rev. D 62, 044020 (2000). gr-qc/0001102. 
[16] N.G. Phillips and B.L. Hu, Int. J. Theor. Phys. 39, 1817 (2000), gr-qc/0004006 Phys.Rev. D 62 , 084017 (2000), gr-qc/0005133.

[17] L. H. Ford and C.-H. Wu, Int. J. Theor. Phys. 42, 15 (2003), gr-qc/0102063.

[18] L. H. Ford and R. Woodard, gr-qc/0411003.

[19] For definitions and derivation of this equation, see, for example, R.M. Wald, General Relativity, (University of Chicago Press, Chicago, 1984) Sect. 9.2.

[20] S.W. Hawking, Commun. Math. Phys. 43, 199 (1975).

[21] K.I. Kellerman and J.M. Moran, Annu. Rev. Astron. Astrophys. 39, 457 (2001).

[22] S.D. Biller et al, Phys. Rev. Lett., 83, 2108 (1999).

[23] Some of the results presented in this appendix are found in textbook presentations, but are included here for completeness. See, for example, M. Ludvigsen, General Relativity, (Cambridge University Press, Cambridge UK, 1999), pp 62 and 106. 